This item was submitted to Loughborough's Research Repository by the author.

Items in Figshare are protected by copyright, with all rights reserved, unless otherwise indicated.

\title{
Energy harvesting from torsional vibrations using a nonlinear oscillator
}

PLEASE CITE THE PUBLISHED VERSION

http://dx.doi.org/10.1115/DETC2016-59467

\section{PUBLISHER}

(c) American Society of Mechanical Engineers (ASME)

\section{VERSION}

AM (Accepted Manuscript)

\section{PUBLISHER STATEMENT}

This work is made available according to the conditions of the Creative Commons Attribution-NonCommercialNoDerivatives 4.0 International (CC BY-NC-ND 4.0) licence. Full details of this licence are available at: https://creativecommons.org/licenses/by-nc-nd/4.0/

\section{LICENCE}

CC BY-NC-ND 4.0

\section{REPOSITORY RECORD}

Gunn, Ben, Panagiotis Alevras, and Stephanos Theodossiades. 2019. "Energy Harvesting from Torsional Vibrations Using a Nonlinear Oscillator". figshare. https://hdl.handle.net/2134/22660. 


\title{
ENERGY HARVESTING FROM TORSIONAL VIBRATIONS USING A NONLINEAR OSCILLATOR
}

\author{
Ben Gunn \\ Wolfson School of Mechanical \& Manufacturing \\ Engineering, Loughborough University, \\ Loughborough, Leicestershire, LE11 3TU, UK
}

\author{
Panagiotis Alevras \\ Wolfson School of Mechanical \& Manufacturing \\ Engineering, Loughborough University, \\ Loughborough, Leicestershire, LE11 3TU, UK
}

\author{
Stephanos Theodossiades \\ Wolfson School of Mechanical \& Manufacturing \\ Engineering, Loughborough University, \\ Loughborough, Leicestershire, LE11 3TU, UK
}

\begin{abstract}
Harvesting ambient energy in a variety of systems and applications is a relatively recent trend, often referred to as Energy Harvesting. This can be typically achieved by harvesting energy (that would otherwise get wasted) through a physical process aiming to convert energy amounts to useful electrical energy. The harvested energy can be thermal, solar, wind, wave or kinetic energy, with the last class mainly referring to harvesting energy from vibrating components or structures. More often these oscillations are error states from the systems' ideal function and through harvesting this potentially wasted energy could be reclaimed and become useful. Regardless of the generally low power output of the devices designed to harvest energy from vibrations, their use remains an attractive concept, which is mostly attributed to the growing use of modern electronic devices that exploit the low power requirements of semi-conductors. Energy Harvesting applications are often met in situations where a network of essential electronic devices, such as sensors in Structural Health Monitoring or bio-implantable devices, becomes hardly accessible. Harvesting ambient vibrations to power up these devices offers the option to utilize wireless sensors rendering these systems autonomous. Typical cases of systems, where ambient vibrations are ubiquitous are met in automotive and aerospace applications. Besides their potentially adverse impact, the energy carried by vibrating parts could be harvested, such that wireless sensors are powered. In this paper, a concept for harvesting torsional vibrations is proposed, based on a concept that employs magnetic levitation to establish a nonlinear Energy Harvester. Experience has shown that linear
\end{abstract}

harvesters require resonant response to operate, often leading to low performance of the device when the excitation frequency deviates from resonance conditions. This is why harvesters with essential nonlinearity are preferred, since they are able to demonstrate high response levels over wider frequency regions. Herein, the conducted study aims to demonstrate the functionality of this concept for torsional systems. A mathematical model of the coupled nonlinear electromechanical system is established, seeking preliminary estimates of the harvested power. The compelling attribute of this system lies in the dependency of its linear natural frequency on the excitation frequency, which is found to cause multiple response peaks in the corresponding frequency spectra. Moreover, the selection of the static equilibrium of the levitating magnet is found to greatly influence the system's response.

\section{INTRODUCTION}

Energy harvesting concerns - amongst others - devices which convert ambient vibration energy into useful electrical energy in a variety of applications in structural, aerospace, and automotive engineering sectors. Quite often, these devices form part of a wireless sensor network in systems where it is impractical to maintain a battery powered device, such as inside car tires [1]. There are three widely employed vibration energy harvesting technologies: piezoelectric, electromagnetic and electrostatic.

Piezoelectric energy harvesters use materials that produce voltage due to applied stress, thus converting the mechanical oscillations to electricity. Piezoelectric ceramics are generally 
very stiff and their brittleness limits their ability to be mass loaded. As such, piezoelectric energy harvesters tend to resonate at high frequencies requiring some means of frequency up-conversion $[2,3]$ to be useful in low frequency applications.

Electrostatic energy harvesters use a variable capacitor with two oppositely charged plates oscillating with respect to each other to drive a current. These energy harvesters require dimension control in a micro-scale, making them well suited to small scale energy harvesters due to their improved performance with decreased plate separation [4].

Electromagnetic energy harvesters use the relative motion between a magnet and a coil to induce electric potential in the coil. This relative motion typically takes the form of an oscillating mass-spring system excited by ambient vibrations. Designs using linear springs, such as those of Shan [5], Beeby [6] and Marin [7], result in a relatively narrow operating frequency range near resonance. Real life applications though call for a wider frequency range operation [8], due to manufacturing tolerances and inconsistent excitation frequencies. To tackle this, it has been proposed to exploit multimodal harvesters where each of the modes would target a different range of operating frequencies. Marin [7] achieved this by combining multiple linear resonators with different natural frequencies to improve the operating range of the harvester.

Another approach to increase the bandwidth is frequency up-conversion, where a low frequency oscillation is used to excite a high frequency system by impacts. An example of such a system was discussed by Zorlu et al [9]. This energy harvester achieved a comparatively good energy density of $190 \mu \mathrm{W} / \mathrm{cm}^{3}$. However, this design has the disadvantage of increased part count, which increases production costs and design complexity.

Mann and Sims [10], Foisal et al [11] and Berdy et al [12] used magnetic levitation, whereas Moss et al [13] used a magnetic tether to create a nonlinear translational energy harvester with a wide operating bandwidth. The main advantage is that the performance of a nonlinear harvester presents relative robustness to variations of the excitation frequency. This harvester also has the advantage of being tunable to a resonant frequency by changing the separation distance of the magnets.

In this paper, magnetic levitation is employed in harvesting energy from rotating components. Numerical integration is used to compute the dynamics of the coupled nonlinear electromechanical system. The influence of key design parameters is investigated, followed by preliminary estimations of the power output.

\section{NOMENCLATURE}

\begin{tabular}{|l|r|}
\hline$A_{c}$ & Cross sectional area of the coil $\left(\mathrm{m}^{2}\right)$ \\
\hline $\mathrm{B}_{\mathrm{r}}$ & Remnant magnetic flux $(\mathrm{T})$ \\
\hline $\mathrm{c}$ & Damping coefficient $(\mathrm{Ns} / \mathrm{m})$ \\
\hline $\mathrm{emf}$ & Electromotive force $(\mathrm{V})$ \\
\hline $\mathrm{F}_{\mathrm{cp}}$ & Centripetal force $(\mathrm{N})$ \\
\hline $\mathrm{F}_{\mathrm{b}}$ & Bottom magnet force $(\mathrm{N})$ \\
\hline $\mathrm{F}_{\mathrm{emf}}$ & Magnetic force generated by the emf $(\mathrm{N})$ \\
\hline
\end{tabular}

\begin{tabular}{|c|c|}
\hline $\mathrm{F}_{\mathrm{m}}$ & Magnet force $(\mathrm{N})$ \\
\hline $\mathrm{F}_{\mathrm{t}}$ & Top magnet force $(\mathrm{N})$ \\
\hline $\mathrm{g}$ & Acceleration due to gravity $\left(9.81 \mathrm{~m} / \mathrm{s}^{2}\right)$ \\
\hline $\mathrm{I}$ & Current (A) \\
\hline $\mathrm{k}$ & Linear stiffness coefficient $(\mathrm{N} / \mathrm{m})$ \\
\hline $\mathrm{k}_{3}$ & Nonlinear stiffness coefficient $\left(\mathrm{N} / \mathrm{m}^{3}\right)$ \\
\hline $\mathrm{L}$ & Inductance $(\mathrm{H})$ \\
\hline $\mathrm{m}$ & Mass of the magnet $(\mathrm{kg})$ \\
\hline $\mathrm{N}_{\mathrm{c}}$ & Number of coil turns \\
\hline $\mathrm{P}$ & Electrical power $(\mathrm{W})$ \\
\hline $\mathrm{P}_{\text {avg }}$ & Average power $(\mathrm{W})$ \\
\hline $\mathrm{R}_{\mathrm{i}}$ & Internal resistance $(\Omega)$ \\
\hline $\mathrm{R}_{1}$ & Load resistance $(\Omega)$ \\
\hline $\mathrm{r}_{1}$ & Inner radius of the coil (m) \\
\hline $\mathrm{r}_{2}$ & Outer radius of the coil $(\mathrm{m})$ \\
\hline rms & Root mean square \\
\hline $\mathrm{V}_{\mathrm{s}}$ & Induced e.m.f. (V) \\
\hline $\mathrm{x}$ & Radial position of the center magnet (m) \\
\hline $\mathrm{X}_{0}$ & Half outer magnet spacing $(\mathrm{m})$ \\
\hline $\mathrm{Z} 1$ & Radial position of the bottom of the coil (m) \\
\hline $\mathrm{Z}_{2}$ & Radial position of the top of the coil (m) \\
\hline $\mathrm{h}_{\text {coil }}=\mathrm{z}_{2}-\mathrm{z}_{1}$ & Radial position of the bottom of the coil (m) \\
\hline$\hat{\Theta}$ & Electromagnetic coupling factor $(\mathrm{Vs} / \mathrm{m})$ \\
\hline$v_{\mathrm{s}}$ & Volume of the magnet $\left(\mathrm{m}^{3}\right)$ \\
\hline$\xi$ & Fill factor \\
\hline$\omega$ & Angular velocity of the shaft (rad/s) \\
\hline$\omega_{0}$ & Constant angular velocity of the shaft (rad/s) \\
\hline$\Omega_{0}$ & Linear resonance frequency $(\mathrm{rad} / \mathrm{s})$ \\
\hline
\end{tabular}

\section{PROBLEM STATEMENT}

The proposed energy harvester consists of a (levitating) magnet that is free to move between two outer (static) magnets that are fixed to the housing of the device, such as to repel the central magnet as shown in FIG. 1. A coil is wrapped around the housing near the oscillating magnet and energy is harvested from the motion of the magnet through the emf induced in the coil. The housing is then attached to a rotating shaft and the whole assembly rotates with the shaft.

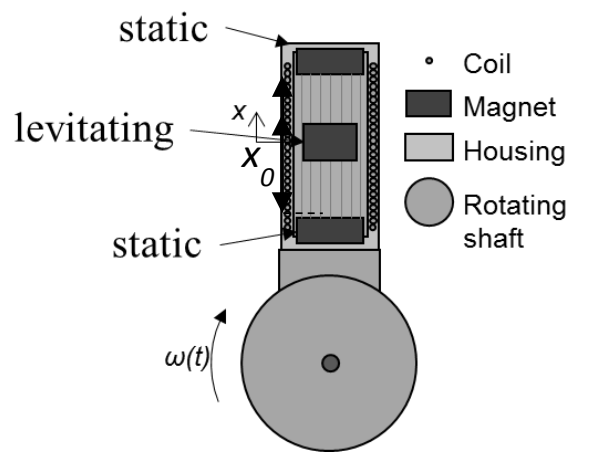

\section{FIG. 1 SKETCH OF THE MAGNETIC LEVITATION HARVESTER}

Mann and Sims [10] demonstrated that the force exerted by the outer magnets on the central magnet can be approximated by a power series of the form: 


$$
F(s)=\sum_{n=0}^{3} \alpha_{n} s
$$

The force between the outer magnets and the levitating one can be calculated as follows:

$$
\begin{aligned}
& F_{b}(x)=\sum_{n=0}^{3} \alpha_{n}\left(x_{0}+x\right)^{n} \\
& F_{t}(x)=\sum_{n=0}^{3} \alpha_{n}\left(x_{0}-x\right)^{n}
\end{aligned}
$$

where $\mathrm{x}_{0}$ is the separation distance between the central magnet's static equilibrium and each of the outer magnets (without considering gravity), whereas $\mathrm{x}$ is the magnet's displacement. Then the net magnetic force reads:

$$
\begin{aligned}
& F_{m}(x)=\left(2 \alpha_{1}+4 x_{0} \alpha_{2}+6 x_{0}^{2} \alpha_{3}\right) x+2 \alpha_{3} x^{3}= \\
& =k x+k_{3} x^{3}
\end{aligned}
$$

From this result, it can be seen that the linear component of the stiffness can be tuned to give a desired resonant frequency by changing the initial separation of the magnets, $\mathrm{x}_{0}$, without affecting the nonlinear stiffness, $\mathrm{k}_{3}$.

The centripetal force acts radially on the central magnet with its magnitude given by:

$$
F_{c p}=m \omega^{2}(r+x)
$$

Taking into account the gravitational force and considering a viscous damping mechanism in the system, the equation of motion of the oscillating magnet is given by:

$$
\begin{aligned}
& m \ddot{x}+c \dot{x}+k x+k_{3} x^{3}= \\
& =m \omega^{2}(r+x)+m g \cos (\omega t)
\end{aligned}
$$

The motion of the magnet induces voltage on the coil according to Faraday's law. This induced voltage is related to the magnet's velocity through the electromagnetic coupling factor, $\hat{\Theta}$, as follows:

$$
V_{s}=\hat{\Theta}(x) \dot{x}
$$

For a magnet passing through the axis of a coil, the coupling factor is given by Mann [10] to be:
$\hat{\Theta}=\left(\frac{N_{c} B_{r} v_{s} \xi}{2 A_{c}}\right) \sum_{i, j=1}^{2}(-1)^{i+j}\left(\ln \left(r_{i}+Z_{i j}\right)-\frac{r_{i}}{Z_{i j}}\right)$

where the fill factor, $\xi$, is the ratio of the coil area occupied by the conductor. $Z_{\mathrm{ij}}$ is given by:

$$
Z_{i j}^{2}=r_{i}^{2}+\left(z_{j}-x\right)^{2}
$$

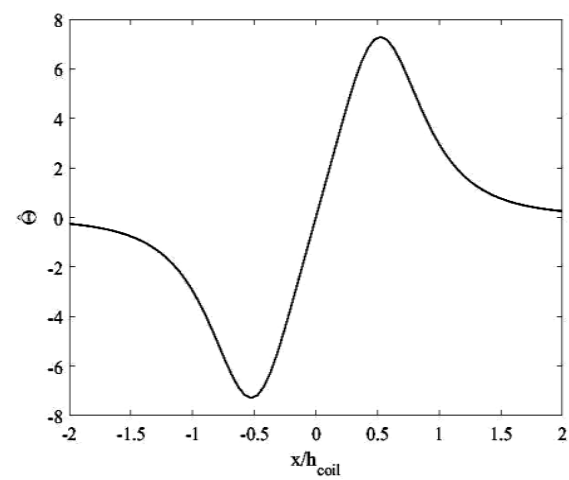

FIG. 2 VARIATION OF THE ELECTROMAGNETIC COUPLING COEFFICIENT ALONG THE AXIS OF THE COIL

$\mathrm{A}_{\mathrm{c}}$ is the coil cross sectional area given by:

$$
A_{c}=\left(r_{2}-r_{1}\right)\left(z_{2}-z_{1}\right)
$$

FIG. 2 shows the coupling factor plotted along the axis of the coil. Note that its extremes are obtained near the two edges of the coil. The current that flows in the coil due to the induced voltage, produces its own magnetic field which, according to Lenz's law, is opposing the magnet's motion by:

$$
F_{\text {emf }}=\hat{\Theta} I
$$

Adding this force in the equation of motion after rearranging Eq. (6), substituting $\Omega_{o}{ }^{2}=k / m$ and $\beta=k_{3} / m$ and considering a constant shaft speed $\omega=\omega_{o}$ gives:

$$
\begin{aligned}
& \ddot{x}+\frac{c}{m} \dot{x}+\left(\Omega_{0}^{2}-\omega_{0}^{2}\right) x+\beta x^{3}-\frac{\hat{\Theta}}{m} I= \\
& =\omega_{0}^{2} r-g \cos \left(\omega_{0} t\right)
\end{aligned}
$$

The coil inductance and internal resistance are acting in series with the load and induced emf, as the schematic of the electric circuit in FIG. 3 shows. Applying Kirchoff's voltage law, the equation for the current flowing through the circuit is given by: 


$$
\dot{I} L+\left(R_{i}+R_{l}\right) I+\hat{\Theta} \dot{x}=0
$$

The instantaneous power delivered to the load can then be calculated by:

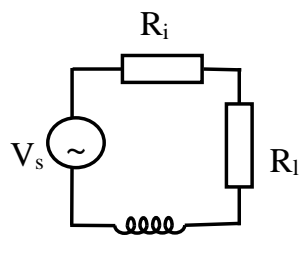

$\mathrm{L}$

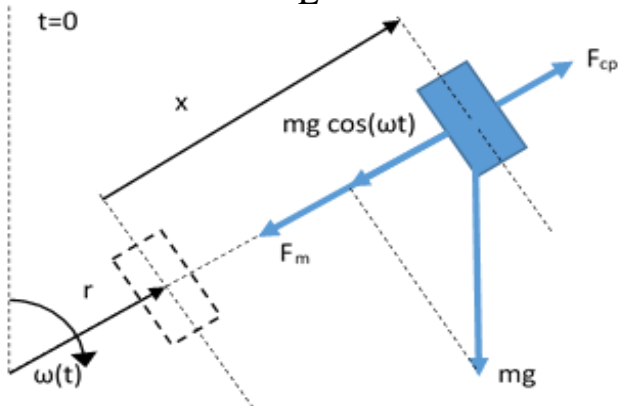

FIG. 3 THE COUPLED ELECTROMECHANICAL SYSTEM

$$
P=I^{2} R_{l}
$$

The average power can be found by integrating Eq. (14) over a cycle of the periodic motion and dividing by the period:

$$
P_{a v g}=\frac{1}{T} \int_{0}^{T} P(t) d t
$$

For maximum power generation, both the coupling factor and the magnet velocity should be at maximum simultaneously [14]. This is why the relative position of the coil with respect to the equilibrium of the magnet has to be carefully chosen.

\section{NUMERICAL RESULTS}

In this section, numerical integration of Eq. (12) is performed for constant shaft angular velocity in order to acquire some insight into the system dynamics. A computational model has been developed in MATLAB $\odot$, accommodating the nonlinear expression of the Electromagnetic Coupling (EC). In what follows, the electromechanical system was simulated for up to 2000 excitation periods, so that steady-state response is established. The parameters of interest were extracted, such as the response velocity amplitude and generated power, using the last 5 periods of the response.

Studying the dynamics of Eq. (12) poses the challenge of interpreting the joint contribution of three forcing components. First, the system is excited by a constant term stemming from the centripetal force acting on the middle magnet. This term comprises the fixed length $r$ (between the centre of the shaft and the static equilibrium point of the middle magnet) and the displacement of the middle magnet, $x$. It is worth noting that $r$ corresponds to the equilibrium when the shaft rests still, i.e. to the point of balance of the net magnetic force which is then the only force acting. Hence, the static equilibrium $r$ depends on the position of the set of static magnets whereas the equilibrium of the magnet's oscillations varies with the shaft's speed. This manipulation gives rise to a constant excitation that depends on the design and the speed of the rotating system; in addition, the variable part contributes to the system's linear frequency through acting against the linear part of the net magnetic force: $\left(\Omega_{o}^{2}-\omega_{o}^{2}\right) x$. In that way, the resulting linear frequency varies along with the shaft speed. Last, a periodic excitation is applied by the gravitational force on the central magnet along the shaft's rotation.

The effect of these three components will be sought individually. First, the distance $r$ is considered in view of the reported influence of the constant excitation in the response of a periodically forced Duffing oscillator [15, 16]. Then, frequency response curves are calculated to capture the influence of the varying linear frequency. Last, the extracted power is calculated assuming the nonlinear coupling between the mechanical and the electromagnetic system, shown in Eq. (8) and a typical set of the coil parameters.

\section{Distance $r$ parametric study}

The influence of the centripetal force is highlighted by varying parameter $r$. Figure 4 shows the velocity response amplitude for a constant excitation frequency $\omega_{0}=90.06 \mathrm{rad} / \mathrm{s}$ and three configurations of the linear stiffness. An interesting observation is that when the net linear restoring force is exactly zero $\left(k=m \omega_{o}^{2}\right)$, a nonlinear jump occurs in the response velocity curve for $r>0.011$. In fact, the maximum value is achieved exactly before the jump occurs. In the same manner, Fig. 4(c) shows the maximum velocity achieved at a similar $r$ value, but with an overall linear behaviour of the system. On the other hand, the case of an exciting frequency greater than $\Omega_{0}$ presents a different trend with the velocity decreasing with increasing $r$, as Fig. 4(a) shows. These figures reveal the existence of a region of $r$ within which the response velocity is extremely high. The significance of these results will be discussed more thoroughly next.

\section{Frequency response curves}

The second part of the numerical analysis is focusing on obtaining frequency response curves of the system's dynamics. Throughout this section, the effect of the system's linear frequency dependency on the excitation frequency is investigated. The linear part of the stiffness is kept constant so that $\Omega_{0}=90.06 \mathrm{rad} / \mathrm{s}\left(f_{0}=14.33 \mathrm{~Hz}\right)$. The distance, $r$, at which the magnetic force is balanced is constant throughout each presented figure, so as to reflect the response of a rotor with varying speed. 


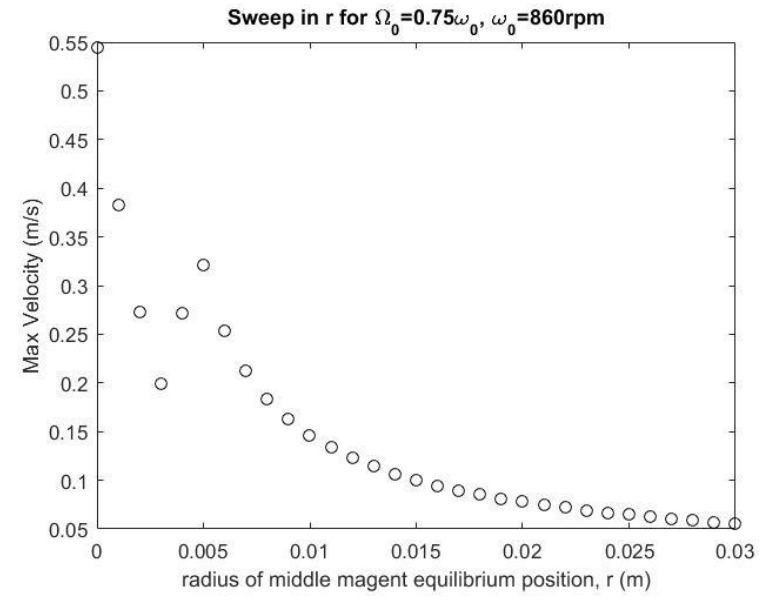

(a)

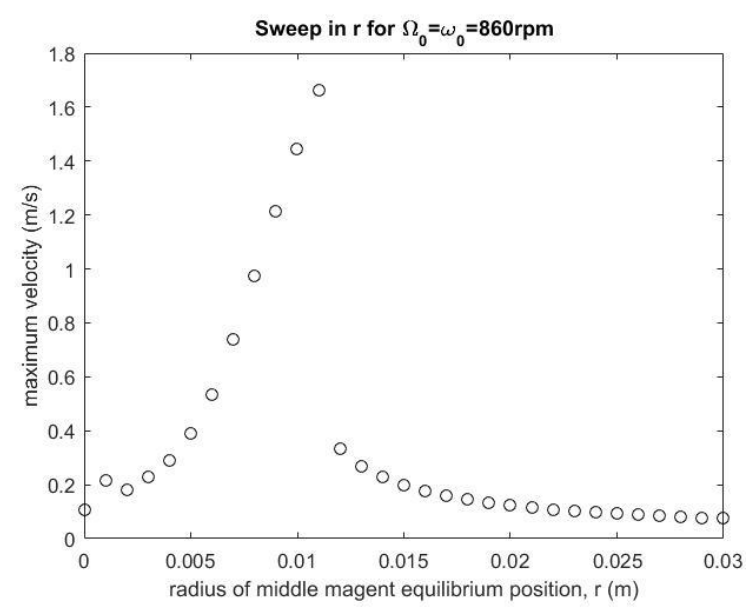

(b)

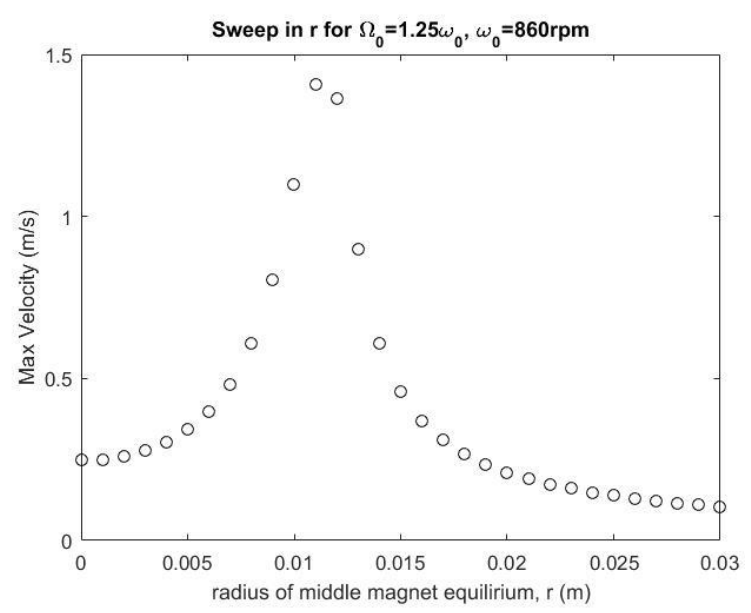

(c)

FIG. 4 PARAMETRIC PLOTS OF VELOCITY RESPONSE AMPLITUDE WITH RESPECT TO $r$ FOR $\zeta=0.03$ AND $\beta=5 \cdot 10^{6}$ $1 / \mathrm{m}^{2} \mathrm{~s}^{2}$.

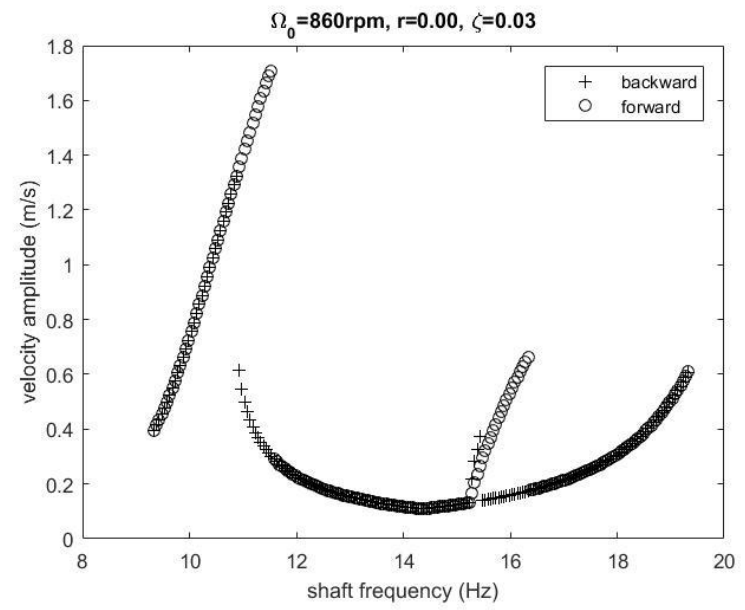

(a)

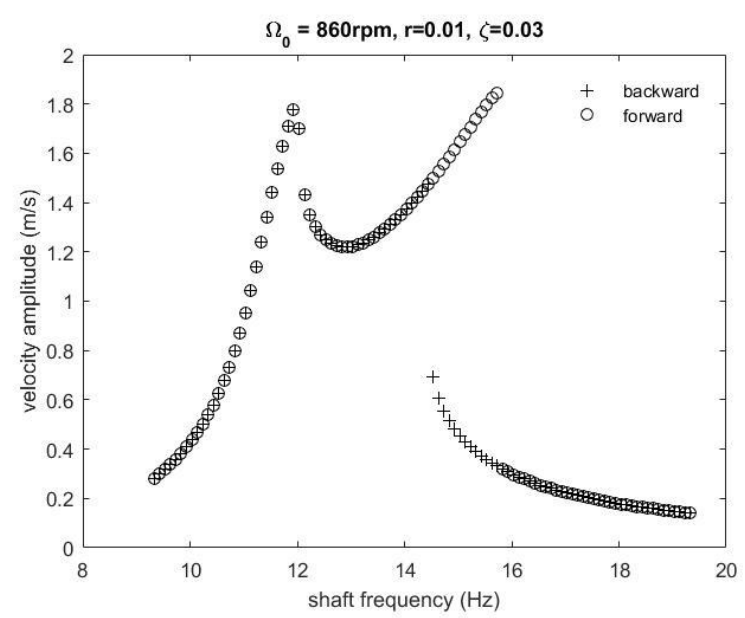

(b)

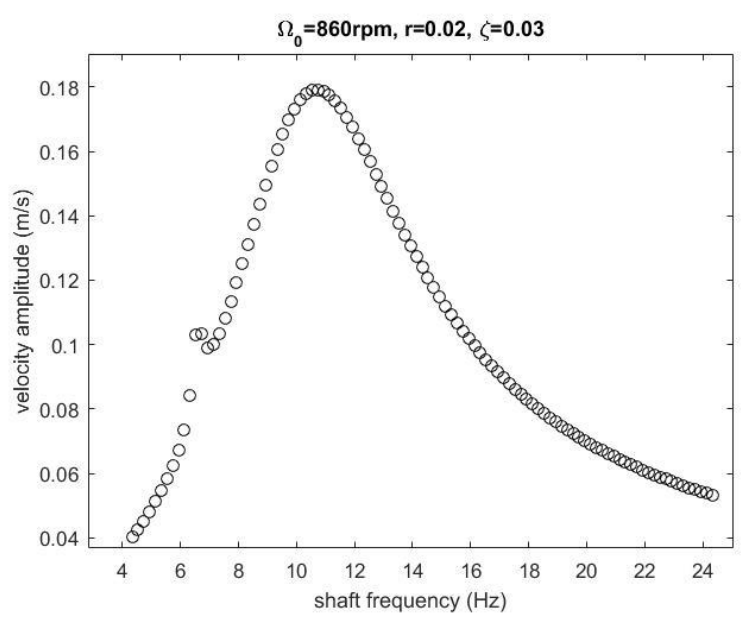

(c)

FIG. 5 FREQUENCY RESPONSE CURVES (VELOCITY AMPLITUDES) FOR $\beta=5 \cdot 10^{6} 1 / \mathrm{m}^{2} \mathrm{~s}^{2}$. 
First, we consider the case that $r=0.0$. Practically, this corresponds to eliminating the centripetal force when the middle magnet rests in equilibrium. In this case, the system dynamics are triggered by the action of gravity. The response is thereafter restored by both the magnetic force and the centripetal force that corresponds to motion $x$. An immediate observation from FIG. 5(a) is that two peaks dominate the response. These peaks are accompanied by equivalent jumps which were identified when sweeping the frequency forward and backwards. Each of these peaks resembles the response of a hardening Duffing oscillator. The attribute of the system shown in Eq. (12) to experience variations of its natural frequency as the excitation frequency changes, gives rise to the aforementioned behaviour. Essentially, when the excitation frequency is lower than that corresponding to the linear part of the magnetic stiffness, i.e. $\omega_{0}<\Omega_{0}$ or $\mathrm{f}_{0}<14.33 \mathrm{~Hz}$ in Fig. 5 , the net linear restoring force is positive. There is a turning point for the sign of the restoring force, that being when $\omega_{0}=\Omega_{0}$. The system is then essentially nonlinear, relying only on the cubic part of the magnetic force to rebound. As soon as $\omega_{0}>\Omega_{0}$, the restoring force becomes negative, practically leading the system away from the equilibrium.

During these changes in the system's dynamics from low excitation frequency to higher, it is possible to acquire at least two solutions that result in an integer frequency ratio. This leads to resonant response of the system with one peak existing for frequencies lower than $\Omega_{0}$ and another for frequencies higher than that.

Another useful observation could be made from the curves shown in Fig. 5, related to the effect of $r$ on the magnitude of the response velocity. Noting that these figures correspond to increasing values of the distance $r=0.0, r=0.01$ and $r=0.02$, it is noted that the highest velocity amplitudes are achieved for the middle case, $r=0.01$. This is consistent with what was extracted from Fig. 4, only that now, the expanded frequency range extends this observation to include the bandwidth of the velocity response. Indeed, in Fig. 5b, the frequency range, for which the velocity is over $1 \mathrm{~m} / \mathrm{s}$ is considerably wide, totalling up to $5 \mathrm{~Hz}$. Complementing the last statement, the amplitude of the velocity in Fig 5c, retracts to rather low values (below 0.2 $\mathrm{m} / \mathrm{s}$ ), reaffirming the deteriorating effect of increasing $r$, with respect to the magnitude of the velocity response.

\section{Time histories of the harvester's response}

A closer view in the system dynamics is obtained by analysing the time histories of the harvester's response. Herein, the time histories of the system's variables - displacement, velocity, current - as well as of the voltage induced on the coil $V_{e}$ and the power of the electric load are presented. These results correspond to a constant excitation frequency $\left(\omega_{0}=\right.$ $90.06 \mathrm{rad} / \mathrm{s}$ ) and $r=0.01 \mathrm{~m}$.

Fig. 6 shows time histories for $\Omega_{0}=0.75 \omega_{0}, \Omega_{0}=\omega_{0}$ and $\Omega_{0}$ $=1.25 \omega_{0}$. In that way, the response is reported for the three distinct cases with respect to the sign of the linear frequency, as they were observed in the previous subsection. The captured history of the current for $\Omega_{0}=\omega_{0}$ and $\Omega_{0}=1.25 \omega_{0}$ shows a second harmonic influencing its profile. In fact, besides the main frequency corresponding to the excitation, $f_{0}=14.33 \mathrm{~Hz}$, the second frequency is measured at $28.64 \mathrm{~Hz}$, revealing the effect of the interaction between the design of the electromagnetic transducer- (mainly the coil) with the oscillating magnet. This is a paramount aspect of the design of an energy harvester of this type, which will be further discussed in the next section.

As it has been mentioned, the harvested power depends on the velocity of the oscillator and the electromagnetic coupling. Thus, one would expect that higher power could be extracted when the response velocity is high. Indeed, a cross-examination of the captured velocity time histories confirms the previous statement, since the recorded power follows the order of the recorded velocity amplitude, with the third case of $\Omega_{0}=1.25 \omega_{0}$ reporting the highest average power of $23.4 \mathrm{~mW}$ (see TABLE 1). This rationale, based on the observations from the time histories, provokes the analysis that follows.

\section{TABLE 1 AVERAGE POWER CORRESPONDING TO THE TIME HISTORIES SHOWN IN FIG. 6.}

\begin{tabular}{|c|c|}
\hline Frequency ratio & Average power $(\mathrm{W})$ \\
\hline$\Omega_{0}=0.75 \omega_{0}$ & 0.00078 \\
\hline$\Omega_{0}=\omega_{0}$ & 0.01454 \\
\hline$\Omega_{0}=1.25 \omega_{0}$ & 0.02339 \\
\hline
\end{tabular}

Since the highest response velocity is sought, then according to FIG. 5, the system having $\mathrm{r}=0.01 \mathrm{~m}$ is considered, where not only does the curve demonstrates the highest velocity amplitudes, but a wider range of frequencies leading to high amplitudes also exists. Repeating the frequency sweeps for this case and considering the coupled response of Eqs. (12) and (13), frequency response curves of the coupled oscillator are obtained.

First, reasonably enough, the response curve retracts to lower velocity values due to the increased damping in the system. Besides the originally considered mechanical damping, the electromagnetic force counteracts the motion of the middle magnet, realizing additional electrical damping in the magnet's motion. This leads to increased overall damping in the response of the oscillator and consequently, the response velocity is expected to be lower than the one shown in FIG. 5.

Calculating the extracted power and averaging over the period of the response, reasonably high power levels up to $43 \mathrm{~mW}$ are observed, as shown in FIG. 7. Additionally, higher values are noted when $\omega_{0}<\Omega_{0}$ (or $f<f_{0}$ ). This is consistent with the time histories, where the highest average power was recorded for a relation between the frequencies abiding to the previous inequality. Also, one should not overlook the bandwidth of the response curve for the power, which approximately conveys that for a $4 \mathrm{~Hz}$ band $(11-15 \mathrm{~Hz})$, the average power will be over $25 \mathrm{~mW}$. 

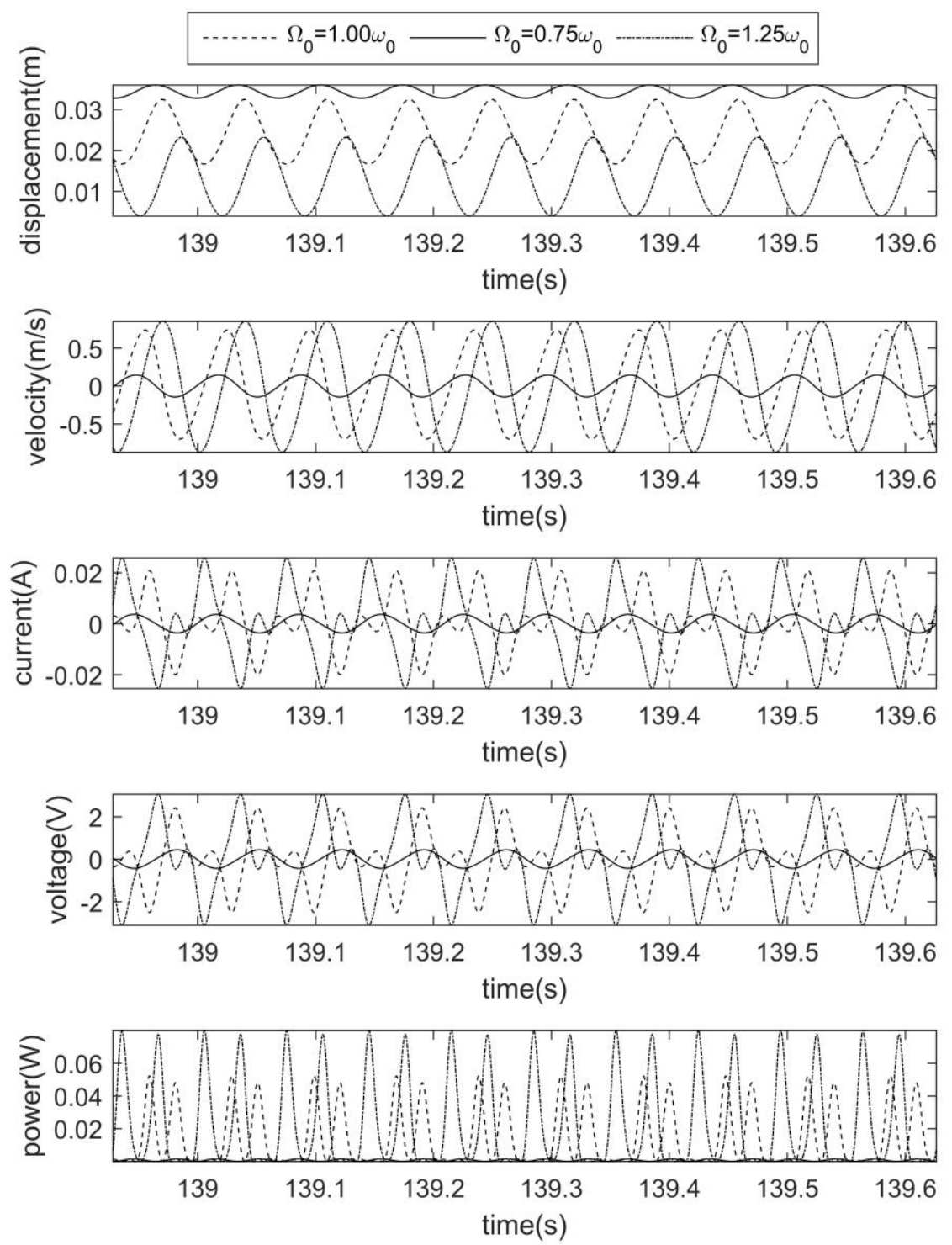

FIG. 6 TIME HISTORIES OF THE SOLUTION TO EQS (12), (13). DASHED LINE SHOWS THE CASE OF $\Omega_{0}=\omega_{0}$, SOLID LINE THE CASE OF $\Omega_{0}=0.75 \omega_{0}$, DASH-DOT LINE THE CASE OF $\Omega_{0}=1.25 \omega_{0}$ AND $\omega_{0}=90.06 \mathrm{rad} / \mathrm{s}, \zeta=0.03, \beta=5 \cdot 10^{6} 1 / \mathrm{m}^{2} \mathrm{~s}^{2}, \mathrm{~m}=0.02 \mathrm{~kg}$ AND THE PARAMETERS IN TABLE 2.

\section{The case of $\omega_{0}=\Omega_{0}$}

A case with particular interest is the one where the energy harvester is essentially nonlinear (in terms of the restoring force). This is achieved by assuming that $\omega_{0}=\Omega_{0}$. Such a system would have the additional advantage of overcoming the need to be tuned to a particular frequency, thus allowing room for harvesting energy from wider frequency ranges. Setting $r=$ $0.01 \mathrm{~m}$, the obtained frequency response curve is shown in FIG. 8. What should be noted is that the velocity amplitude acquires rather high values for a significant frequency band. In particular, the velocity is over $1 \mathrm{~m} / \mathrm{s}$ almost for the entire examined frequency range. Further on, it is over $1.3 \mathrm{~m} / \mathrm{s}$ for a range of about $9 \mathrm{~Hz}(8-17 \mathrm{~Hz})$. This result demonstrates the potential of nonlinear energy harvesters in overcoming the issue of tuning that is inherent in linear or weakly nonlinear systems.

TABLE 2 PARAMETERS OF THE COIL USED IN THE SIMULATIONS.

\begin{tabular}{|r|r|}
\hline Inductance $\mathrm{L}(\mathrm{mH})$ & 8 \\
\hline Number of coil turns $\mathrm{N}_{\mathrm{c}}$ & 1500 \\
\hline Resistance to inductance ratio $\rho(\mathrm{Ohms} / \mathrm{H})$ & 15000 \\
\hline Radius of the coil $\mathrm{r}_{1}(\mathrm{~mm})$ & 9 \\
\hline Radius of the coil $\mathrm{r}_{2}(\mathrm{~mm})$ & 12 \\
\hline Coil height $\mathrm{h}(\mathrm{mm})$ & 19 \\
\hline Volume of the magnet $v_{\mathrm{s}}\left(\mathrm{m}^{3}\right)$ & $2.67 \cdot 10^{-6}$ \\
\hline Fill factor $\xi$ & 0.33 \\
\hline Remnant magnetic flux $\mathrm{B}_{\mathrm{r}}(\mathrm{T})$ & 1.31 \\
\hline
\end{tabular}




\section{DISCUSSION}

In this paper, a concept for energy harvesting from rotating mechanical systems (similar to those met in aerospace and automotive powertrain applications) is proposed. The concept is based on magnetic levitation. A set of static magnets is considered, with another magnet free to vibrate between them. The pair of the static magnets is housed in an attachment that is considered to be rigidly mounted on a rotating shaft, with the axis connecting the three magnets extending radially. The aim of this paper is to examine the dynamics of this system via numerical simulations, setting the ground work for the proofof-concept of this design.

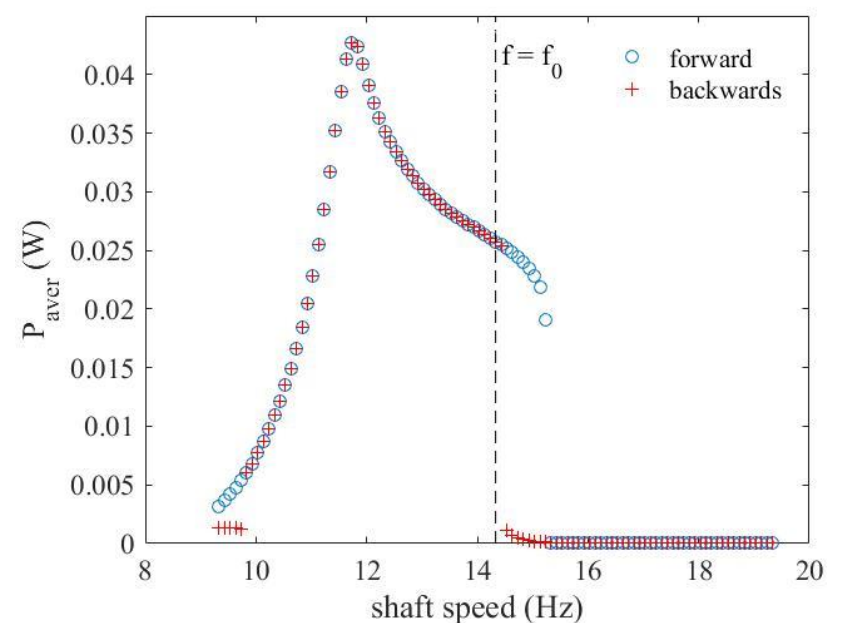

FIG. 7 AVERAGE POWER OF THE COUPLED ELECTROMECHANICAL SYSTEM FOR THE SAME PARAMETERS AS IN FIG. 5B AND THE PARAMETERS SHOWN IN TABLE 2.

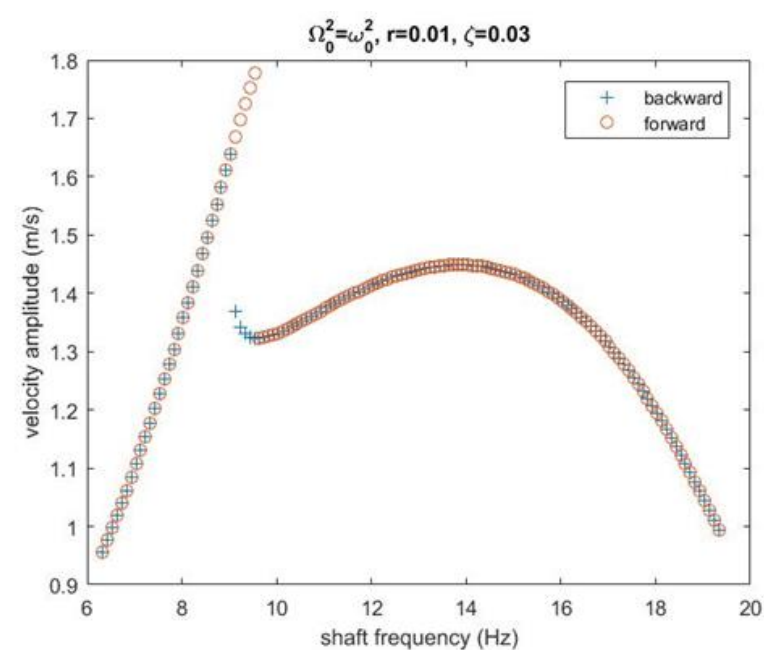

FIG. 8 FREQUENCY RESPONSE CURVE FOR AN ESSENTIALLY NONLINEAR ENERGY HARVESTER (COUPLED ELECTROMECHANICAL SYSTEM)

In this preliminary study, the speed of the rotor is considered to be constant. This was deemed as necessary for an early-stage analysis due to the complicated dynamics of the system, even after this simplification is applied. The main attribute that leads to a complex response is the dependency of the system's linear frequency on the excitation frequency. This means that during a typical frequency sweep several interesting phenomena arise, including nonlinear jumps and multiplicity of the backbone curve i.e. there is not just a single hardening or softening curve, but rather a combination of them. This can lead, given a proper design, to a rather wideband frequency response curve. The importance of such a quality in a harvester's response is the robustness of the extracted power against changes in the main frequency of the vibrations driving the device. Especially in the case that the design is essentially nonlinear, the system can be detached from a preferential frequency and offers the potential of even greater robustness of the response velocity (or else the power).

The system is exposed to constant excitation, stemming from the centripetal force acting on the levitating magnet. A design parameter, related to the magnitude of the centripetal force, is the distance $r$ from the centre of the shaft to the magnet's equilibrium. The numerical integration showed that there is a threshold for this distance, above which the response velocity is confined to ever decreasing amplitudes. The physical interpretation of this observation is that the centripetal force is pushing the levitating magnet to oscillate closer to the top magnet. Given the nonlinear nature of the magnetic force, the middle magnet is trapped within a potential well of low velocity, thus deteriorating the harvesting capabilities of this device. Yet, it has been observed that in certain cases (see FIG. $4 \mathrm{~b}$ for example), this happens with a nonlinear jump.

The second crucial variable besides the velocity is the electromagnetic coupling, the values of which depend on the position of the magnet along the coil's axis. This means that apart from the selection of a coil that maximizes the EC, one has to consider the exact time history of the middle magnet's displacement, including the position of the new equilibrium. Imagine a case that the new equilibrium is several times the amplitude away from the edge of the coil. The extracted power would then be negligible regardless of the velocity and the values of the EC in the coil. Moreover, as one could notice in the time histories of Fig. 6, the current contains a second harmonic frequency along with the main one. This can be seen in the induced voltage as well and is caused due to the asymmetry of the EC around its edge, so that when the equilibrium point of the oscillations (with an amplitude comparable to the coil's length) is offset from the centre of the coil, the variation of the EC gives rise to a second harmonic. These observations point out the significance of considering the optimum positioning of the coil during the design process, in order to avoid intervals of low power output within the harvester's cycle.

\section{ACKNOWLEDGMENTS}

The authors wish to express their gratitude to the EPSRC for the financial support extended to the "Targeted energy transfer in powertrains to reduce vibration-induced energy 
losses" Grant (EP/L019426/1), under which this research was carried out.

\section{REFERENCES}

[1] K. Bharat Singh, V. Bedekar, S. Taheri and S. Priya, "Piezoelectric vibration energy harvesting system with an adaptive frequency tuning mechanism for intelligent tires," Mechatronics, vol. 22, no. 7, pp. 970-988, 2012.

[2] J. Rastegar, C. Pereira and H.-L. Nguyen, "PiezoelectricBased Power Sources for Harvesting Energy from Platforms with Low Frequency Vibration," Smart Structures and Materials, vol. 6171, p. 617101, 2006.

[3] C. Viñolo, D. Toma, A. Mànuel and J. del Rio, "Sea motion electrical energy generator for low-power applications," in OCEANS, MTS/IEEE, Bergen, 2013.

[4] S. Boisseau, G. Despesse and B. Ahmed Seddik, "SmallScale Energy Harvesting," in Electrostatic Conversion for Vibration Energy Harvesting, Grenoble, France, InTech, 2012.

[5] X. Shan, Z. Xu and T. Xie, "New electromechanical coupling model and optimisation of an electromagnetic energy harvester," Ferroelectrics, vol. 450, no. 1, pp. 6673, 2013.

[6] S. Beeby, R. Torah and M. J. Tudor, "A micro electromagnetic generator for vibration energy harvesting," Micromechanics and microengineering, vol. 17, no. 7, 2007.

[7] A. Marin, S. Bressers and S. Priya, "Multiple cell configuration electromagnetic vibration energy harvester," Journal of Physics D: Applied Physics, vol. 44, no. 29, pp. 295501-295512, 2011.

[8] N. Elvin and A. Erturk, Advances in energy harvesting methods, New York: Springer Science \& Business Media, 2013.

[9] O. Zorlu, E. T. Topal and H. Kulah, "A vibration-based electromagnetic energy harvester using mechanical frequency up-conversion method," IEEE Sensors Journal, vol. 11, no. 2, pp. 481-488, 2011.

[10] B. P. Mann and N. D. Sims, "Energy harvesting from the noonlinear oscillations of magnetic levitation," Journal of sound and vibration, vol. 329, no. 9, pp. 515-530, 2009.

[11] A. R. M. Foisal, C. Hong and G. S. Chung, " Multifrequency electromagnetic energy harvester using a magnetic spring cantilever," Sensors and actuators, vol. 182, pp. 106-113, 2012.

[12] D. Berdy, D. Valentino and D. Peroulis, " Kinetic energy harvesting from human walking and running using a magnetic levitation energy harvester," Sensors and actuators, vol. 222, p. 262-271, 2015.

[13] S. Moss, G. Hart, S. Burke and P. Carman, "Hybrid rotarytranslational vibration energy harvester using cycloidal motion as a mechanical amplifier," Applied Physics Letters, vol. 104, no. 3, 2014.

[14] B. A. Owens and B. P. Mann, "Linear and nonlinear coupling models in vibration-based energy harvesting," Journal of Sound and Vibration, vol. 331, no. 4, pp. 922937, 2012.

[15] I. Kovacic, M. J. Brennan and B. Lineton, "On the resonance response of an asymmetric Duffing oscillator," International Journal of Non-Linear Mechanics, vol. 43, no. 9, pp. 858-867, 2008.

[16] S. Theodossiades and S. Natsiavas, "Non-linear dynamics of gear-pair systems with periodic stiffness and backlash," Journal of Sound and vibration, vol. 229, no. 2, pp. 287310, 2000. 